\title{
Le Panthéon renversé. À propos de la mort de Bergotte et de la mort de Swann
}

\author{
Yan Hamel \\ TÉLUQ
}

Le "grand homme » est l'une des icônes sacrées que vénère la III République. Dix ans après l'arrivée de Thiers au pouvoir, le régime « a besoin d'une mythologie refondatrice de l'État, soutenu par les vertus civiques. Le décès de Victor Hugo va fournir l'occasion d'une célébration civile unissant le peuple et son gouvernement dans la ferveur républicaine. » (Degraene, 2005, p.17) Héritière des commémorations instaurées par la Première République, cette dévotion réveillée prend à nouveau pour cadre l'église Sainte-Geneviève, qui, encore une fois, est dé-consacrée ${ }^{1}$ et

1 En 1830, l'église Sainte-Geneviève a été retirée une première fois du culte catholique. Elle retrouvera ensuite ses fonctions liturgiques sous le Second Empire. 
re-panthéonisée en vue de servir à la panthéonisation de ceux dont la mémoire sera éternellement conservée par la "patrie reconnaissante ». L'importance, pour la symbolique et l'imaginaire républicains, du rituel de transfert des restes illustres vers le tombeau néoclassique du Vearrondissement n'est plus à démontrer: le Panthéon est le premier des trois monuments analysés dans Les Lieux de mémoire (Ozouf, 1997²); les historiens des idées, des mentalités et de l'architecture s'entendent à peu près tous pour reconnaître que l'œuvre de Soufflot s'inscrit «parmi les symboles fondateurs de la République Française » et que ce statut a été conservé dans la longue durée après « maints avatars » (Deming, 1989, p. 97). On ne suscitera assurément aucune controverse en donnant raison à Jean-Claude Bonnet, pour qui l'institution du Panthéon et le culte des grands hommes font « encore partie aujourd'hui de [l'] horizon culturel [français] et de la mémoire vivante d'une nation à la fois littéraire et républicaine » (1998, p. 13).

\section{Le Panthéon républicain}

Cela dit, à la Belle Époque, l'importance et la signification du Panthéon ne sont pas aussi largement et aussi sereinement reconnues. En instaurant le culte nouveau, le pouvoir entend réaliser rien de moins qu'une révolution mémorielle. Changeant de régime politique, la France, dorénavant laïque et démocratique, doit se doter de nouvelles valeurs et trouver,

\footnotetext{
2 Les deux autres monuments consacrés par le collectif de Pierre Nora sont la mairie, analysée par Maurice Agulhon, et les monuments aux morts, analysés par Antoine Prost.
} 
tant bien que mal, une nouvelle cohésion nationale. Pour ce faire, il lui faut imposer à aussi large échelle que possible une mémoire nationale unitaire. Le pouvoir doit repenser le mémorable, redéfinir ce qui, du passé, sera conservé et transmis, en (re)donnant aux événements et aux hommes d'autrefois un sens éthique propre à conforter et à pérenniser la République. Clairement dirigé contre les monarchistes, les catholiques, les bonapartistes, les anarchistes et tous les autres adversaires du régime, le "grand homme » tel qu'il s'implante alors et tel qu'il a perduré jusqu'à nos jours fut engendré par cet impératif politique et social :

[On] n'accepte plus désormais que la valeur dépende du bon plaisir du monarque ou de la préséance d'un monde séparé. Elle doit s'afficher comme une vertu évidente et contagieuse qui soit susceptible d'être acquise par tous, au moyen d'une nouvelle exemplarité et d'une nouvelle pédagogie héroïque. Le moment est venu d'universaliser la gloire en la dissociant d'un honneur exclusif qui se présente comme la passion et la vanité d'une seule classe sociale. (Bonnet, 1998, p. 32)

Avec la panthéonisation de Victor Hugo et celles qui l'ont suivie - Sadi Carnot, Émile Zola, Léon Gambetta, Jean Jaurès, etc. -, la République redéfinit ce qu'est la grandeur humaine véritable en promouvant le rappel cérémoniel de certains morts choisis pour être intronisés, comme le dit Mona Ozouf, "précepteurs du genre humain» (1997, p.161). Voilà une revenance institutionnalisée des morts rendus illustres dont les ambitions cardinales et la signification profonde avaient, à l'époque, été explicitées par Edgar Quinet: la transformation de l'église Sainte-Geneviève en un Panthéon visait à réaliser « le beau rêve de la Constituante, [à savoir] l'éducation morale d'un peuple dans la liberté par le souvenir consacré des meilleurs » (1883, p 157). 
La République veut (ré)unifier la population - «un peuple» - grâce à l'enseignement de valeurs et de principes essentiellement bourgeois: c'est ce qu'il faut entendre par «l'éducation morale » qui se réalise « dans la liberté ». Comme le remarque l'historien de l'architecture Mark K. Deming, le «civisme» est ainsi «institué nouvelle religion dominante.» (1989, p. 101) Il s'agit en bonne partie, non pas d'éradiquer la mémoire de la France religieuse, mais plutôt de la détourner au profit de l'imagerie et de la symbolique républicaine : le « souvenir consacré» évoqué par Quinet est conservé par une église primitivement dédiée à Sainte-Geneviève, au sein de laquelle les fresques hagiographiques de Puvis de Chavannes encadrent des monuments célébrant, notamment, Mirabeau et la Convention nationale. Sous la voute du Panthéon et dans sa crypte, une mémoire d'une paradoxale sacralité laïque vise à transmettre le souvenir de quelques vies idéales qui ont incarné mieux que les autres un ensemble de valeurs données à la fois pour universellement humaines et pour typiquement républicaines : c'est le souvenir, affirme Quinet, « des meilleurs ».

Se devant d'être unitaire, cette mémoire de la République doit aussi savoir se perpétuer, inchangée, de génération en génération. Quinet écrit encore que le Panthéon, ce « temple de la renommée » (1883, p. 141), allait être «l'âme éternellement vivante de la France. Les morts y trouveraient leur récompense et les vivants leur voie vers l'avenir. À l'entrée de la Révolution se dressait ce grand phare éclatant de la mort pour éclairer le chemin. » (1883, p. 145) Cimetière « conçu comme une terre d'émulation » (Ozouf, 1997, p. 161), le Panthéon est ce par quoi le pouvoir républicain assure la continuité entre les disparus exemplaires et la postérité, c'est-à-dire entre les morts et ceux qui sont encore à naître. Il faut y voir l'aboutissement d'un 
changement de paradigme qui s'est réalisé sur une durée relativement longue, trouvant son origine au tournant du XVIII ${ }^{\mathrm{e}}$ siècle, comme l'ont montré les travaux de Philippe Ariès :

Culte privé donc, mais aussi dès l'origine, culte public. Le culte du souvenir s'est tout de suite étendu de l'individu à la société, à la suite d'un même mouvement de la sensibilité. Les auteurs de projets de cimetière du XVIII siècle souhaitent que les cimetières soient à la fois des parcs organisés pour la visite familiale, et aussi des musées d'hommes illustres, comme la cathédrale Saint-Paul à Londres. Les tombes des héros et des grands hommes y seraient vénérées par l'État. C'est une conception différente de celle des chapelles ou des caveaux dynastiques, comme Saint-Denis, Westminster, l'Escorial ou les Capucins de Vienne. Une représentation nouvelle de la société naît en cette fin du XVIIIe siècle, qui se développera au XIX'e, et qui trouvera son expression dans le positivisme d'Auguste Comte, forme savante du nationalisme. On pense, et même on sent, que la société est composée à la fois des morts et des vivants, et que les morts sont aussi significatifs et nécessaires que les vivants. La cité des morts est l'envers de la société des vivants, ou, plutôt que l'envers, son image, et son image intemporelle. Car les morts ont passé le moment du changement et leurs monuments sont les signes visibles de la pérennité de la cité. (Ariès, 1975, p. 60-61)

Une fois venu à terme, ce long mûrissement est toutefois gommé par l'idéologie républicaine triomphante. Il n'est pas conservé en mémoire comme le seront les grands hommes érigés au rang de souvenirs impérissables précieusement conservés par la nation. Bien au contraire: à la fin du XIXe siècle, «le Panthéon est le lieu d'une Révolution installée, éternisée, triomphante, qui a oublié, ou voulu oublier, son histoire. » (Ozouf, 1997, p. 168) La panthéonisation est non seulement la cérémonie par laquelle un mort est rappelé à la vie de la Cité, mais aussi la cérémonie par laquelle chacun des morts successivement honorés par la Cité réaffirme la valeur, 
en le réincarnant par-delà le trépas, du civisme bourgeois mis à l'avant-plan par le régime. Celui-ci assure par là le maintien d'un idéal inaltéré, inaltérable, dans une société qui entend ellemême se conserver, inaltérée et inaltérable.

Voilà pourquoi le Panthéon peut être tenu pour l'exemple républicain par excellence «d'un art propagandiste et éducateur » (Ozouf, 1997, p. 175) : « Ce qui soutient le culte des grands hommes, au moment où il s'élabore, c'est la foi dans la solidarité spontanée de l'esthétique et de la morale, dans la nécessaire docilité du public à la leçon des sens et dans l'efficacité d'un art-pédagogue. » (Ozouf, 1997, p. 175) Bien qu'il faille donner globalement raison à Mona Ozouf, il serait plus juste de définir le Panthéon, non comme un art-pédagogue, mais plutôt comme une alliance d'arts pédagogiques réunissant l'architecture, la peinture, la sculpture, la performance théâtrale, sans oublier la littérature, qui est exploitée de multiples façons par ce complexe dispositif mémoriel. En plus des discours de panthéonisation, qui sont autant de morceaux d'éloquence rendant hommage à l'air du temps, de la devise gratifiante qui se donne à lire sur le fronton de l'édifice et des multiples inscriptions qui ornent murs et tombeaux, les Belles Lettres sont le seul des arts qui soit panthéonisé en la personne de plusieurs " grands hommes » conservés dans la crypte. Avec les politiciens et les scientifiques, les écrivains comptent en effet pour beaucoup dans la cohorte de ceux qui habitent les caveaux de la montagne Sainte-Geneviève, ce qui n'est pas le cas des peintres, des sculpteurs ou des musiciens. Et, puisque prime l'utilitarisme éducatif de l'art-pédagogue, les écrivains glorifiés sont moins retenus pour la beauté ou l'originalité esthétique souvent indéniable - de leur œuvre, que pour leur contribution au progrès de la société, au triomphe de la justice 
et de la liberté, en somme à la défense et à l'illustration des valeurs et des institutions démocratiques-républicaines. À l'évidence, «J'accuse » a davantage compté que Les RougonMacquart dans la décision de porter les restes de Zola au Panthéon le 20 novembre 1906. En effet, le sénateur Boissy d'Anglas déclare à la chambre, pour justifier la proposition de panthéoniser le chef de file du naturalisme : «Ce n'est pas pour son mérite littéraire, d'ailleurs immense, que semblable proposition vous est faite. C'est uniquement pour un acte de courage civique, grand et tragique comme les circonstances au milieu desquelles il s'accomplit. » (cité dans Drouin, 2008, p. 2526) Par les écrivains qui y sont consacrés, et par les raisons politiques motivant cette consécration, le Panthéon est une forme de manifeste national pour une littérature progressiste et démocratique, en prise directe sur les affaires de la Cité.

\section{Le «grand homme » selon À la recherche du temps perdu}

Ce qui précède ne nous conduit pas à Marcel Proust (qu'on imagine mal être panthéonisé), et encore moins à la Recherche du temps perdu ou aux morts de Bergotte et de Swann qu'annonce pourtant le sous-titre du présent article. Le lecteur intéressé par cette œuvre et par ces personnages serait tout à fait en droit de se questionner sur la pertinence d'un tel état des lieux, synthétisant l'essentiel de ce que l'on peut dire sur l'idéologie mémorielle mise à l'avant plan par « l'École normale des morts» (cité dans Ozouf, 1997, p. 1743), alors que ce monument aussi froid que pompier n'a strictement rien à voir

\footnotetext{
${ }^{3}$ Le mot est d’André Billy.
} 
avec le roman proustien. Le lecteur ordinaire qui a déjà parcouru le cycle sait que le XVIIIe siècle et son héritage y sont fort peu présents. Le spécialiste consciencieux qui se donnerait la peine d'effectuer une enquête lexicographique exhaustive serait de son côté en droit d'ajouter que le mot "Panthéon » n'est pas utilisé une seule fois dans les milliers de pages qui composent la Recherche ${ }^{4}$. L'un et l'autre pourraient du reste objecter avec raison que le chef-d'œuvre proustien est une cathédrale gothique en prose (Jonson, 1975; Fraisse, 1990), et non pas l'«hommage réactionnaire de Soufflot aux cathédrales » (Ozouf, 1997, p. 165).

À ces protestations, il faut répondre que le propos du présent article n'est pas de chercher à minimiser les différences opposant le rapport aux morts illustres qu'instaurent, chacun à leur manière, le texte proustien et le Panthéon: c'est au contraire leur opposition radicale qui fera sens, étant entendu que le premier peut se situer par ce qui le différencie du second. Alors que la question de la mort chez Proust a déjà été abondamment analysée par la critique (Le Roux-Kieken, 2005, p. 15), prendre le Panthéon comme contre-modèle mémoriel de la Recherche devrait éclairer sous un jour neuf le traitement que l'écriture proustienne réserve au souvenir des morts, en en élucidant un aspect méconnu : la force de la critique éthique, esthétique et politique qu'il oppose à l'idéologie et à la mémoire nationale républicaines.

Les spécialistes qui se sont intéressés aux figures du trépas chez Proust divisent les morts de la Recherche en trois

\footnotetext{
4 J'ai accompli une telle enquête grâce au moteur de recherche « The ARTFL Project », conçu et mis en ligne par le Département de langues et littératures romanes de l'Université de Chicago, <http://artfl-project.uchicago.edu>.
} 
catégories : «la mort en énigme », « la mort en sourdine » et « la mort en vitrine » (Henrot, 2004, p. 652). Dans la perspective privilégiée par le présent article, il n'y aurait à peu près rien à dire de la première catégorie, qui masque la mort, et de la deuxième, qui la passe quasiment sous silence. Il en va en revanche tout autrement pour la «mort en vitrine » : lorsqu'elle ne concerne pas un personnage attaché au narrateur par des liens amoureux, affectifs ou familiaux, comme c'est par exemple le cas pour la grand-mère, cette "mise en vitrine » de la mort par l'écriture proustienne est, dans le cycle romanesque, un équivalent textuel de la panthéonisation, qui est une «mise en vitrine » républicaine de la mort. Les trépassés mis à l'avantplan par le roman proustien se voient conférer une valeur sociale et esthétique; ils acquièrent une exemplarité qui en fait, comme le montrera l'analyse de deux cas représentatifs, un envers proustien du rituel funéraire mis à l'honneur par la IIIe République.

Swann et Bergotte sont deux des personnages qui initient le narrateur au monde des arts et de la littérature. Remplissant tous deux une fonction pédagogique, guidant le narrateur dans le parcours qui le mène à la découverte de sa vocation, ils sont pour lui des manières de précepteurs. Étant donné que Bergotte et Swann sont aussi fortement opposés dans le schéma actantiel du personnel romanesque - tandis que le premier incarne la figure du grand écrivain, le second, qui ne crée rien, ne dépasse jamais le statut de dilettante brillant mais stérile - , les significations que revêtent leurs morts respectives sont complémentaires. Cette complémentarité sémantique est structurellement accentuée par le fait que les deux épisodes sont quasiment juxtaposés, étant situés à moins de dix pages d'intervalle, au centre de La Prisonnière. 
Par le traitement qu'ils réservent à la mort, les deux passages voisins traitent de revenance; ils explorent la porosité entre l'irréversibilité de la mort individuelle et la continuité transindividuelle des mémoires assurant la perpétuation sociale de ces morts par-delà le trépas. Et cette exploration est éminemment signifiante d'un point de vue intertextuel et sociosémantique : les deux passages érigent le retour commémoratif de la mort à la vie en un nœud textuel de tensions où s'entremêlent et se heurtent des pratiques discursives associées à la platitude journalistique, au snobisme aristocratique et mondain, à la religiosité catholique, au spiritisme fin de siècle, au rationalisme comtien ainsi qu'à diverses philosophies esthétiques.

L'éloge funèbre tel qu'on le publiait dans les feuilles de l'époque apparaît dans les deux passages pour être disqualifié. Bergotte, qui meurt dans une exposition de peinture hollandaise, craint in extremis d'être le fait divers du lendemain. Cette appréhension rapportée par le narrateur oppose la superficialité stéréotypée du compte rendu signé par un publiciste au sens profondément mystique et spirituel que l'écriture proustienne, on le verra, confère à la mort de l'écrivain. La critique du point de vue typique que le journal adopte sur la mort est encore plus explicite dans le passage consacré à la fin de Swann, où est enchâssé le pastiche d'un article du Gaulois rendant hommage au disparu. Le narrateur rapporte que, lisant le journal,

son annonce [de la mort de Swann] m'avait arrêté net, comme tracée en mystérieuses lignes inopportunément interpolées. Elles avaient suffi à faire d'un vivant quelqu'un qui ne peut plus répondre à ce qu'on lui dit, un nom, un nom écrit, passé tout à coup du monde réel dans le royaume du silence. C'est elles qui 
me donnaient encore maintenant le désir de mieux connaître la demeure où avaient autrefois résidé les Verdurin et où Swann, qui alors n'était pas seulement quelques lettres tracées dans un journal, avait si souvent dîné avec Odette. (Proust, 1988c, p. 705)

Ces lignes évoquent les premières recherches conduisant à la rédaction d'Un amour de Swann et des autres parties du cycle où le personnage entre en scène. Le journal marque la mort, l'interruption du discours et du dialogue, il est annonciateur de disparition, de non-signifiance éternisée (c'est « le royaume du silence ») que le narrateur veut empêcher et dépasser, d'abord pour lui-même, mais aussi pour celui à qui il s'adresse. Du côté de chez Swann et les autres tomes de la Recherche redonneront vie au personnage en des centaines et des milliers de lignes qui conféreront une tout autre épaisseur et une tout autre durée mémorielle à son existence révolue.

Le grand aristocrate, dont la vie se continue par-delà la mort grâce à la perpétuation du titre, est un autre contremodèle, présent seulement, celui-là, dans le passage consacré à Swann: «Sans doute c'est d'une façon anonyme, sans distinction d'individualité, qu'on demeure le duc d'Uzès. Mais la couronne ducale en tient quelque temps ensemble les éléments. » (Proust, 1988c, p. 704) Sort peu enviable toutefois dans la mesure où ce modèle commémoratif ne préserve en rien le souvenir des réalisations individuelles; seul le titre nobiliaire subsiste par-delà la succession de ceux qui l'ont porté, vouant par le fait même à un oubli rapide les vies menées ainsi que les réalisations accomplies par chacun d'entre eux. À cela s'ajoute encore que, en régime démocratique, la couronne elle-même est donnée pour périssable : la survie mémorielle de ceux qui l'ont portée et qui, ce faisant, l'ont constituée en un être transgénérationnel se trouve sous la garde d'une caste en 
délitement qui ne parvient plus à préserver ses souvenirs sur la longue durée, ce que montre entre autres le passage du «bal de têtes », à la fin du Temps retrouvé, où l'amnésie générale qui affecte le Faubourg Saint-Germain rend cette société inapte à départager les aristocrates véritables des parvenus.

Toute partielle et défaillante soit-elle, la mémoire aristocratique dépasse néanmoins de loin en durabilité le souvenir socialement conservé des «bourgeois ultramondains » qui, « aussitôt [...] morts, se désagrègent et fondent, "démoulés" » (Proust, 1988c, p. 705). De toutes les formes de renommée envisagées par le texte proustien, le succès mondain est celle qui assure à son détenteur la viabilité post mortem la plus courte, comme le montre, toujours dans le passage consacré à la mort de Swann, l'évocation de Cartier, qui fut un proche de Mme de Guermantes, le meilleur ami du duc de La Trémoïlle et l'un des hommes les plus brillants de France. Après sa mort, il devient « quelque chose de si informe qu'on le grandirait presque en l'apparentant au bijoutier Cartier, avec lequel il eût souri que des ignorants pussent le confondre! » (Proust, 1988c, p. 705) S'il faut en croire la Recherche, les souvenirs de la productivité bourgeoise sont, sous la IIIe République, mieux ancrés collectivement, et plus durables, que ceux de l'éminence mondaine.

Paradoxalement, c'est l'œuvre proustienne qui, en les critiquant, contribue à perpétuer le souvenir quasiment perdu des journaux, des aristocrates et des brillants mondains de la Belle Époque, allant ainsi à rebours de la crise mémorielle qui afflige la modernité en général et la IIIe République en particulier (Terdiman, 1993). Mais, ce faisant, la Recherche réhabilite moins une mémoire collective journalistique, 
aristocratique ou mondaine qu'elle n'érige la littérature au rang de monument pérenne devant assurer, sur la longue durée, la survivance sociale de certains morts exemplaires. Pour parvenir à pérenniser la mémoire de ces morts mis en vitrine, la Recherche procède notamment à un détournement de l'imagerie religieuse, qui n'est pas tout à fait étrangère à celui que réalisait, en ces mêmes années, le Panthéon, tout se passant comme si, en ces années de laïcité triomphante, l'héritage catholique continuait à être seul en mesure de fournir les matériaux discursifs et iconographiques susceptible de mettre en place une représentation de la grandeur humaine véritable : le passage consacré à la mort de Bergotte transfigure littéralement les œuvres publiées par l'auteur. Celles-ci sont des intermédiaires entre l'ici-bas et le monde supraterrestre qui assurent le triomphe christique du mort sur sa propre disparition: "toute la nuit funèbre, aux vitrines éclairées, ses livres, disposés trois par trois, veillaient comme des anges aux ailes éployées et semblaient pour celui qui n'était plus, le symbole de sa résurrection" (Proust, 1988c, p. 693). Il faut mentionner au passage que la Recherche gratifie aussi l'écrivain fictif d'une résurrection littérale, puisque, grâce à une erreur de composition qui échappa à Proust, Bergotte est l'un des 14 personnages morts qui réapparaissent dans le roman: il revient, dans Albertine disparue, donner son avis sur un article que le narrateur a publié dans Le Figaro.

Dans le cas de Swann, qui n'a pas laissé d'œuvre littéraire, ce sont les tomes de la Recherche parus avant La Prisonnière qui assurent cette revenance et cette perpétuation du mort dans la mémoire sociale. Le narrateur, en une sortie hors des frontières de la fiction digne du Quichotte, se donne en effet le pouvoir de considérer la réception qu'ont reçue les premiers tomes du 
cycle romanesque dont il est pourtant l'un des personnages. Ce passage, extraordinaire dans l'économie du roman, rend poreuses, non seulement les frontières entre la mort et la vie, mais aussi celles qui devraient séparer la fiction et la réalité sociale. Ici, le discours intradiégétique du narrateur et les volumes extradiégétiques d'À la recherche du temps perdu assurent conjointement le retour au monde des vivants de Swann trépassé et de la principale «clé » historique servant de modèle au personnage, Charles Haas, qui est présent sur le tableau évoqué par le narrateur :

Et pourtant, cher Charles Swann, que j'ai si peu connu quand j'étais encore si jeune et vous près du tombeau, c'est déjà parce que celui que vous deviez considérer comme un petit imbécile a fait de vous le héros d'un de ses romans, qu'on recommence à parler de vous et que peut-être vous vivrez. Si dans le tableau de Tissot représentant le balcon du Cercle de la rue Royale, où vous êtes entre Galliffet, Edmond de Polignac et Saint-Maurice, on parle tant de vous, c'est parce qu'on voit qu'il y a quelques traits de vous dans le personnage de Swann. (Proust, 1988c, p. 705)

Ces lignes laissent également entendre que le roman proustien dispose d'un pouvoir de reviviscence et de transcendance plus fort que la toile de Tissot, laquelle n'aurait pu, à elle seule, assurer à Charles Haas une célébrité équivalente à celle dont jouit Swann. On pourrait ajouter que, dans ce cas particulier, le roman contribue à faire (sur)vivre le tableau et son peintre; Le Cercle de la rue Royale fut, en avril 2011, acquis par le Musée d'Orsay pour la modique somme de 4 millions d'euros, en bonne partie parce qu'il est mentionné par le narrateur de la Recherche et que, dans la figure de Charles Haas, la plupart des visiteurs cultivés voient une représentation de Charles Swann. 
Les qualités de ce dernier comme les qualités de Bergotte, et comme les qualités des œuvres littéraires assurant la survie post mortem de ces deux illustres personnages, n'ont rien à voir avec ce qui est digne d'être honoré par la mémoire républicaine et son Panthéon. Plusieurs passages autoréflexifs de la Recherche montrent que les ambitions esthétiques et philosophiques $\mathrm{du}$ roman proustien sont radicalement étrangères à toute forme de projet civique ou d'engagement politique républicain :

Je sentais que je n'aurais pas à m'embarrasser des diverses théories littéraires qui m'avaient un moment troublé notamment celles que la critique avait développées au moment de l'affaire Dreyfus et avait reprises pendant la guerre, et qui tendaient à "faire sortir l'artiste de sa tour d'ivoire", et à traiter des sujets non frivoles ni sentimentaux, mais peignant de grands mouvements ouvriers, et, à défaut de foules, à tout le moins non plus d'insignifiants oisifs («j'avoue que la peinture de ces inutiles m'indiffère assez », disait Bloch), mais de nobles intellectuels, ou des héros. (Proust, 1988d, p. 460)

L'écriture proustienne ne préserve pas la mémoire de Swann, ce dreyfusard mou, réfractaire à toute forme de grandiloquence démocratique, pour des raisons valables d'un point de vue républicain, tout comme la Recherche elle-même ne reste pas à la mémoire lettrée pour des raisons de ce type. De son côté, Bergotte est explicitement disqualifié d'un point de vue politique, moral et civique : dans la première partie d'À l'ombre des jeunes filles en fleur, le diplomate Norpois méprise chez cet auteur "toutes ces chinoiseries de forme, toutes ces subtilités de mandarin déliquescent» (Proust, 1988a, p. 465). Mais à l'inverse, du point de vue défendu par le narrateur, c'est justement cette intransitivité de l'art, cet hyper-raffinement de l'écriture, qui est en mesure de sauver en ramenant le mort à la 
vie. Les pages relatant les circonstances dans lesquelles Bergotte meurt exposent dans son absurdité la plus grotesque l'inutilité morale, sociale ou politique de l'art véritable. L'auteur est terrassé par le bas corporel : ce sont en effet une crise d'urémie et une indigestion de pommes de terre mal cuites qui en viennent à bout (Proust, 1988c, p. 692). Il trouve en outre la mort parce qu'il a voulu contempler une dernière fois la Vue de Delft de Ver Meer, et ce, uniquement pour admirer un petit pan de mur jaune qui lui avait jusqu'alors échappé et qui avait été signalé à son attention par un critique (nous retrouvons encore ici le journal mortifère). Or, ce petit pan de mur jaune est la métonymie parfaite d'un art intransitif, autosuffisant, irrécupérable par quelque principe ou cause autre que luimême. La reprise du motif chinois, qui motivait le rejet exprimé par Norpois deux mille pages plus tôt, ne dit pas ici autre chose : le «petit pan de mur jaune [...] était si bien peint qu'il était, si on le regardait seul, comme une précieuse œuvre d'art chinoise, d'une beauté qui se suffirait à elle-même » (Proust, 1988c, p. 692). Ce fragment de tableau est, pour Bergotte mourant, un idéal qu'il a échoué à atteindre, ce qui place son œuvre (et sa vie) en situation d'infériorité par rapport à la peinture (et à la vie du peintre) : «C'est ainsi que j'aurais dû écrire, disait-il. Mes derniers livres sont trop secs, il aurait fallu passer plusieurs couches de couleur, rendre ma phrase en ellemême précieuse, comme ce petit pan de mur jaune. » (Proust, 1988c, p. 692) À cela s'ajoute que le jaune de ce petit pan de mur est une couleur grotesque, du moins si on en juge par le traitement qui lui est réservé par l'écriture proustienne (Bergotte ne meurt pas par hasard d'urémie). L'œuvre de Vermeer est plus explicitement encore rendue absurde et 
ridicule par la place qu'elle occupe dans les ultimes pensées, informes et séniles, d'un artiste diminué en train de mourir :

Dans une céleste balance lui apparaissait, chargeant l'un des plateaux, sa propre vie, tandis que l'autre contenait le petit pan de mur si bien peint en jaune. Il sentait qu'il avait imprudemment donné la première pour le second. [...] Il se répétait : «Petit pan de mur jaune avec un auvent, petit pan de mur jaune. » Cependant il s'abattit sur un canapé circulaire [...]. Un nouveau coup l'abattit, il roula du canapé par terre où accoururent tous les visiteurs et gardiens. Il était mort. (Proust, 1988c, p. 692)

Cependant, dans un mouvement proprement hugolien, ce grotesque mortifère et noir - ou plutôt jaune - de la scène est immédiatement et diamétralement renversé vers le sublime. La suite du passage convoque sans solution de continuité des images et des représentations proches du spiritisme fin de siècle, auquel croyait d'ailleurs Bergotte qui, nous apprend un autre passage d'Albertine disparue, faisait tourner les tables. La fin des considérations à propos de cette mort sont ainsi tirées vers une forme d'élévation supraterrestre :

Mort à jamais? Qui peut le dire? Certes, les expériences spirites pas plus que les dogmes religieux n'apportent de preuve que l'âme subsiste. Ce qu'on peut dire, c'est que tout se passe dans notre vie comme si nous y entrions avec le faix d'obligations contractées dans une vie antérieure; il n'y a aucune raison dans nos conditions de vie sur cette terre pour que nous nous croyions obligés à faire le bien, à être délicats, même à être polis, ni pour l'artiste athée à ce qu'il se croie obligé de recommencer vingt fois un morceau dont l'admiration qu'il excitera importera peu à son corps mangé par les vers, comme le pan de mur jaune que peignit avec tant de science et de raffinement un artiste à jamais inconnu, à peine identifié sous le nom de Ver Meer. Toutes ces obligations qui n'ont pas leur sanction dans la vie présente semblent appartenir à un monde différent, fondé sur la bonté, le scrupule, le sacrifice, un monde 
entièrement différent de celui-ci, et dont nous sortons pour naître à cette terre, avant peut-être d'y retourner, revivre sous l'empire de ces lois inconnues auxquelles nous avons obéi parce que nous en portions l'enseignement en nous, sans savoir qui les y avait tracées, ces lois dont tout travail profond de l'intelligence nous rapproche et qui sont invisibles seulement - et encore! - pour les sots. De sorte que l'idée que Bergotte n'était pas mort à jamais est sans invraisemblance. (Proust, 1988c, p. 693)

Ce passage pourrait être l'objet d'une analyse très étendue. De façon à respecter les limites imparties à la présente étude, il faudra limiter sa lecture critique à deux remarques. D'une part, les qualités supraterrestres mises en lumière par l'existence de l'œuvre d'art - «la bonté, le scrupule, le sacrifice» — sont aussi exaltées par la République et son Panthéon, à cette différence de taille près que l'idéologie républicaine, tout en les exaltant et en soulignant leur caractère exceptionnel, ne les situe pas moins au niveau terrestre de l'accomplissement social et politique. L'écriture proustienne s'emploie d'autre part à rendre le supraterrestre crédible aux yeux d'un narrataire rationaliste, a-religieux, qui est réfractaire au surnaturel et enclin à douter qu'il puisse y avoir quelque forme que ce soit de vie outre-vie. C'est ce que présupposent les phrases interrogatives « Mort à jamais? Qui peut le dire? », de même que la concession «Certes, les expériences spirites pas plus que les dogmes religieux n'apportent de preuve que l'âme subsiste ». Le texte devient polémique; il cherche, contre un adversaire explicitement mis en scène, à s'approprier les valeurs humaines et artistiques véritables tout en définissant le lieu où elles se situent et le moyen de les atteindre.

La mort de Swann accorde elle aussi une grande importance au surnaturel, qui prend la forme d'une allégorie. 
Celle-ci n'est pas grotesque - esthétique dévolue par Proust à la représentation réaliste de la mort triviale - , mais tend plutôt vers une forme de macabre héritée du Moyen Âge. Le début de l'extrait anthropomorphise une mort qui se situe au-delà de nos capacités sensorielles et qui est rendue perceptible par les procédés métaphoriques de l'écriture :

La mort de Swann! Swann ne joue pas dans cette phrase le rôle d'un simple génitif. J'entends par là la mort particulière, la mort envoyée par le destin au service de Swann. Car nous disons la mort pour simplifier, mais il y en a presque autant que de personnes. (Proust, 1988c, p. 703)

Nous ne possédons pas de sens qui nous permette de voir, courant à toute vitesse, dans toutes les directions, les morts, les morts actives dirigées par le destin vers tel ou tel. Souvent ce sont des morts qui ne seront entièrement libérées de leur tâche que deux, trois ans après. (Proust, 1988c, p. 704)

Il faut ajouter qu'une autre forme de merveilleux est mise à l'avant-plan, celle-là, par une série d'incartades narratives. Nombre de considérations amènent le narrateur à déroger de sa position au sein de l'histoire et du récit pour devenir soit narrateur omniscient, soit instance extradiégétique. On a déjà vu que Swann est tiré par l'écriture du côté de Charles Haas tel que représenté par Tissot. Le tout se déroule dans une adresse directe et simultanée au personnage de roman et au modèle du peintre, confondus, et qui sont tous les deux morts, l'écriture niant symboliquement l'impossibilité de la communication avec les trépassés. L'autre passage rapporte les dernières pensées de Bergotte qui, n'ayant pas été verbalisées par le personnage, ne devraient pas être accessibles à un narrateur intradiégétique. On pourrait voir ces écarts au pacte de lecture et à l'esthétique réaliste proustienne comme des fautes de composition, mais on peut aussi les lire comme une marque du pouvoir que l'écriture de la 
Recherche s'auto-confère, celui d'établir un passage simultané entre la fiction et la réalité, entre la vie et ce qui outrepasse la vie. Indépendamment de tout civisme, de tout impact politique direct sur les affaires de la cité républicaine, la forme romanesque élaborée par Proust ramène les morts historiques à la vie fictive, et inversement, pour qu'ils reviennent, de générations en générations, à la mémoire sociale des lecteurs.

Pour Mona Ozouf, celui qui est l'auteur d'un rapport de panthéonisation est un «donateur de gloire» (1997, p. 171). Le narrateur de la Recherche est lui aussi un donateur de gloire, mais il s'agit dans son cas d'une gloire particulière qu'il a le pouvoir de conférer individuellement, par la force d'un art intransitif et, pour cette raison même, incivique. Se dépeignant sous les traits d'un artiste réfractaire à toute forme d'engagement, le narrateur du Temps retrouvé n'en cherche pas moins à rallier ses lecteur dans un combat esthétique et social qui l'oppose à l'héritage de la Première République : « N'imitons pas les révolutionnaires qui par "civisme" méprisaient, s'ils ne les détruisaient pas, les œuvres de Watteau et de La Tour, peintres qui honorent davantage la France que tous ceux de la Révolution.» (Proust, 1988d, p.467) Faire d'individus tels que Bergotte et Swann les grands hommes rappelés de la mort à la vie est, pour l'écriture proustienne, une autre manière de résister à l'implantation d'une mémoire nationale républicaine en transmettant une mémoire collective parallèle - la collectivité étant ici celle que forment le narrateur et son narrataire. Cette mémoire pérenne transmise aux lecteurs postulés de la Recherche affirme une vision de la grandeur et, avec elle, la valeur de principes esthétiques et éthiques qui, pour être supraterrestres, sont fondamentalement a-républicains. 


\section{Bibliographie}

ARIÈS, Philippe. (1975), Essais sur l'histoire de la mort en Occident du Moyen Âge à nos jours, Paris, Seuil, coll. «Points ».

BonneT, Jean-Claude. (1998), Naissance du Panthéon. Essai sur le culte des grands hommes, Paris, Fayard, coll. « L'esprit de la cité ».

DEgRAEnE, Jean-François. (2005), Petit Dictionnaire des grands hommes du Panthéon, Paris, Éditions du patrimoine.

DEMING, Mark K. (1989), « Le Panthéon révolutionnaire », dans Le Panthéon, symbole des révolutions. De l'Église de la Nation au Temple des grands hommes, Montréal / Paris, Centre Canadien d'Architecture / Caisse nationale des monuments historiques et des sites / Picard éditeur.

Drouin, Michel. (2008), Zola au Panthéon : la quatrième affaire Dreyfus, Paris, Perrin.

FrAISSE, Luc. (1990), L'OEuvre cathédrale. Proust et l'architecture médiévale, Paris, José Corti, 1990.

HEnRoT, Geneviève. (2004) " Mort », dans Annick Bouillaguet et Brian G. Rogers (dir.), Dictionnaire Marcel Proust, Paris, Honoré Champion, p. 652-655.

IFRI, Pascal. (1983), Proust et son narrataire dans À la recherche du temps perdu, Genève, Droz, coll. «Histoire des idées et critique littéraire ».

Jonson, Theodore Jr. (1975), «Proust et l'architecture : considérations sur le problème du roman-cathédrale», 
Bulletin de la Société des Amis de Marcel Proust et des Amis de Combray, no 25, p. 16-34.

LE ROUX-KIEKEN, Aude. (2005), Imaginaire et écriture de la mort dans l'œuvre de Marcel Proust, Paris, Honoré Champion.

Ozouf, Mona. (1997 [1984]), « Le Panthéon », dans Pierre Nora (dir.), Les Lieux de mémoire 1. La République, Paris, Gallimard, coll. «Quarto », p. 155-178.

Proust, Marcel. (1988), À la recherche du temps perdu I, Paris, Gallimard, coll. « Bibliothèque de la Pléiade ».

—. (1988), À la recherche du temps perdu II, Paris, Gallimard, coll. « Bibliothèque de la Pléiade ».

—. (1988), À la recherche du temps perdu III, Paris, Gallimard, coll. « Bibliothèque de la Pléiade ».

—. (1988), À la recherche du temps perdu IV, Paris, Gallimard, coll. « Bibliothèque de la Pléiade».

QuinET, Edgar. (1883), « Le Panthéon », dans Eugène-Emmanuel

Viollet-le-Duc et Edgar Quinet, Les Églises de Paris Le Panthéon, Paris, C. Marpon et E. Flammarion.

Terdiman, Richard. (1993), Present Past: Modernity and the Memory Crisis, Ithaca, Cornell University Press.

\section{Résumé}

Cet article montre que le rituel républicain de la panthéonisation peut être tenu pour un contre-modèle 
mémoriel d'À la recherche du temps perdu de Marcel Proust. Analysant la mort de Bergotte et la mort de Swann, deux extraits du roman La Prisonnière, l'auteur analyse comment l'œuvre proustienne travaille à transmettre des modèles de "grands hommes» et une mémoire collective parallèle aux modèles et à la mémoire nationale. Par cette forme implicite d'engagement dans et par l'écriture, le cycle romanesque affirme et perpétue la valeur de principes esthétiques qui sont fondamentalement a-républicains.

\begin{abstract}
This article shows that the republican ritual of pantheonisation can be considered as a memorial counter-model of Marcel Proust's Recherche du temps perdu. The author analyses the death of Bergotte and the death of Swann, two excerpts of the novel La Prisonnière, and demonstrates that the Recherche passes on models of great men and a collective memory that are parallel to republican models and to national memory. With this form of implicit engagement in and by writing, the novel cycle maintains and perpetuates the value of aesthetic principles that are fundamentally non-republican.
\end{abstract}

